\title{
A RECIPROCAL RELATION FOR HERMITE POLYNOMIALS
}

\author{
Christopher S. Withers and Saralees Nadarajah \\ (Received 28 February, 2021)
}

Abstract. For $x \in \mathbb{R}$, the ordinary Hermite polynomial $H_{k}(x)$ can be written

$$
H_{k}(x)=\mathbb{E}\left[(x+\mathrm{i} N)^{k}\right]=\sum_{j=0}^{k}\left(\begin{array}{c}
k \\
j
\end{array}\right) x^{k-j} \mathrm{i}^{j} \mathbb{E}\left[N^{j}\right],
$$

where $\mathrm{i}=\sqrt{-1}$ and $N$ is a unit normal random variable. We prove the reciprocal relation

$$
x^{k}=\sum_{j=0}^{k}\left(\begin{array}{l}
k \\
j
\end{array}\right) H_{k-j}(x) \mathbb{E}\left[N^{j}\right] .
$$

A similar result is given for the multivariate Hermite polynomial.

\section{Introduction}

By Withers $[\mathbf{1 0}]$, for $x \in \mathbb{R}$, the ordinary Hermite polynomial $H_{k}(x)$ can be written

$$
H_{k}(x)=\mathbb{E}\left[(x+\mathrm{i} N)^{k}\right]=\sum_{j=0}^{k}\left(\begin{array}{l}
k \\
j
\end{array}\right) x^{k-j} \mathrm{i}^{j} \mathbb{E}\left[N^{j}\right],
$$

where $\mathrm{i}=\sqrt{-1}$ and $N$ is a unit normal random variable. This result and its multivariate analog [10] have received useful applications in several areas: seasonal modeling of multivariate distributions of Metocean parameters in marine operations [6]; expansions for multivariate diffusions [1]; stochastic response of non-linear oscillation system under random excitation [16]; an algorithm for computing the multivariate Faa di Bruno's formula [3].

The aim of this short note is to prove the reciprocal relation

$$
x^{k}=\sum_{j=0}^{k}\left(\begin{array}{l}
k \\
j
\end{array}\right) H_{k-j}(x) \mathbb{E}\left[N^{j}\right]
$$

and its multivariate analog. That is, $H_{k}(x), x^{j}$ can be replaced by $x^{k}, H_{j}(x)$ if all signs are made positive. This allows any polynomial in $x$ to be easily written as a linear combination of $H_{j}(x)$.

For multivariate moments, cumulants and Hermite polynomials, there are two notations in use. For example, for $\mathbf{X} \in \mathbb{R}^{p}$, a random vector, one can denote $\mathbb{E}\left[X_{1} X_{p}\right]$ by $m_{1,0, \ldots, 0, p}$ or by $m^{1, p}$ and $\mathbb{E}\left[X_{1} X_{3}^{2}\right]$ by $m_{1,0,2, \ldots, 0}$ or by $m^{1,3,3}$. We shall call them the sub form and the super form. The sub form has to deal with

2010 AMS Mathematics Subject Classification: 33F99.

Key words and phrases: Hermite polynomial; Multivariate normal distribution; Normal distribution. 
the zeros but that is easy for $p=2$ or 3 . Let $\mathbb{N}$ be the non-negative integers. For $\mathbf{x} \in \mathbb{R}^{p}$ and $\mathbf{k}, \mathbf{j} \in \mathbb{N}^{p}$, set

$$
\mathbf{x}^{\mathbf{k}}=x_{1}^{k_{1}} \cdots x_{p}^{k_{p}}, \mathbf{k} !=k_{1} ! \cdots k_{p} !, \text { and }\left(\begin{array}{c}
\mathbf{k} \\
\mathbf{j}
\end{array}\right)=\frac{\mathbf{k} !}{\mathbf{j} !(\mathbf{k}-\mathbf{j}) !} .
$$

Then the sub form of the moment is $m_{\mathbf{k}}=\mathbb{E}\left[\mathbf{X}^{\mathbf{k}}\right]$, and similarly for cumulants and Hermite polynomials. It is used, for example, by Stuart and Ord [9], Withers [10], and for Charlier-Edgeworth expansions for the simpler case of a sample mean, by Bhattacharya and Ghosh $[\mathbf{2}]$ and Withers and Nadarajah [15].

For the super forms, suppose that $j_{1}, \ldots, j_{r} \in \mathbb{N}$ and $k \leq r$. We use the shorthand notation $k-r$ for a suffix $j_{k}, \ldots, j_{r}$. Set

$$
x_{1-r}=x_{j_{1}, \ldots, j_{r}}=x_{j_{1}} \cdots x_{j_{r}} .
$$

The super form of the general moment of $\mathbf{X} \in \mathbb{R}^{p}$ is

$$
m^{1-r}=m^{j_{1}, \ldots, j_{r}}=\mathbb{E}\left[X_{j_{1}} \cdots X_{j_{r}}\right]=\mathbb{E}\left[X_{1-r}\right],
$$

and similarly for cumulants and Hermite polynomials.

Now suppose that $\mathbf{X} \sim \mathcal{N}_{p}\left(\mathbf{0}_{p}, \mathbf{V}\right)$, a normal random $p$-vector with zero means, covariance $\mathbf{V}$, and density $\phi_{\mathbf{V}}(\mathbf{x})$ say. For $\mathbf{x} \in \mathbb{R}^{p}$, set

$$
\mathbf{y}=\mathbf{V}^{-1} \mathbf{x}, \mathbf{Y}=\mathbf{V}^{-1} \mathbf{X} \sim \mathcal{N}_{p}\left(\mathbf{0}_{p}, \mathbf{V}^{-1}\right),\left(V^{j, k}\right)=\mathbf{V}^{-1} .
$$

In shorthand notation, we set

$$
V^{1-r}=V^{j_{1}, \ldots, j_{r}}=\mathbb{E}\left[Y_{1-r}\right]=\sum_{\left(\begin{array}{c}
p \\
r
\end{array}\right)} V^{j_{1}, j_{2}} \cdots V^{j_{r-1}, j_{r}}
$$

for $r$ even, where we use the convention that for $f_{1-r}=f_{j_{1}, \ldots, j_{r}}, \sum_{\left(\begin{array}{c}p \\ r\end{array}\right)} f_{1-r}$ sums over all $\left(\begin{array}{l}p \\ r\end{array}\right)$ permutations of $j_{1}, \ldots, j_{r}$ giving distinct terms.

Set $\partial_{\mathbf{x}}=\partial / \partial \mathbf{x}$. The sub form of the multivariate Hermite polynomial is

$$
\begin{aligned}
H_{\mathbf{k}} & =H_{\mathbf{k}}(\mathbf{x}, \mathbf{V})=\phi_{\mathbf{V}}(\mathbf{x})^{-1}\left(-\partial_{\mathbf{x}}\right)^{\mathbf{k}} \phi_{\mathbf{V}}(\mathbf{x}) \\
& =\mathbb{E}\left[(\mathbf{y}+\mathrm{i} \mathbf{Y})^{\mathbf{k}}\right]=\sum_{\mathbf{0}_{p} \leq \mathbf{j} \leq \mathbf{k}}\left(\begin{array}{c}
\mathbf{k} \\
\mathbf{j}
\end{array}\right) \mathbf{y}^{\mathbf{k}-\mathbf{j}} \mathbb{E}\left[(\mathrm{i} \mathbf{Y})^{\mathbf{j}}\right]
\end{aligned}
$$

by [10]. For example, if $p=2$,

$$
H_{k_{1}, k_{2}}=\mathbb{E}\left[(\mathbf{y}+\mathrm{i} \mathbf{Y})_{1}^{k_{1}}(\mathbf{y}+\mathrm{i} \mathbf{Y})_{2}^{k_{2}}\right]=\sum\left(\begin{array}{c}
k_{1} \\
j_{1}
\end{array}\right)\left(\begin{array}{c}
k_{2} \\
j_{2}
\end{array}\right) y_{1}^{k_{1}-j_{1}} y_{2}^{k_{2}-j_{2}} \mathbb{E}\left[\left(\mathrm{i} Y_{1}\right)^{j_{1}}\left(\mathrm{i} Y_{2}\right)^{j_{2}}\right]
$$

summed over $0 \leq j_{1} \leq k_{1}, 0 \leq j_{2} \leq k_{2}$. Its super form is

$$
\begin{aligned}
H^{1-r} & =H^{j_{1}, \ldots, j_{r}}(\mathbf{x}, \mathbf{V})=\phi_{\mathbf{V}}(\mathbf{x})^{-1}\left(-\partial_{x_{j_{1}}}\right) \cdots\left(-\partial_{x_{j_{r}}}\right) \phi_{\mathbf{V}}(\mathbf{x})=\mathbb{E}\left[\prod_{k=1}^{r}(\mathbf{y}+\mathrm{i} \mathbf{Y})_{j_{k}}\right] \\
& =\sum_{0 \leq s \leq r / 2}(-1)^{s} \sum_{\left(\begin{array}{c}
r \\
s
\end{array}\right)} y_{1-(r-2 s)} V^{(r-2 s+1)-r} \\
& =y_{1-r}-\sum_{\left(\begin{array}{c}
r \\
2
\end{array}\right)} y_{1-(r-2)} V^{(r-1)-r}+\sum_{\left(\begin{array}{c}
r \\
4
\end{array}\right)} y_{1-(r-4)} V^{(r-3)-r}-\cdots
\end{aligned}
$$


and follows from (1.2).

As we saw for $p=2$, the use of (1.2) is more messy. We could write say $H^{1-1}$ rather than $H^{1}$ for $H^{j_{1}}$ but this seems pedantic as the context makes it clear whether $H^{j_{1}}$ is meant. See Section 2 of [10] for their bi-orthogonality and [15] for their application to the multivariate Charlier differential series. Both these use the sub forms.

Section 2 contains the main result, namely Equation (2.2). Section 3 introduces the multivariate modified Hermite polynomial. Possible extensions are discussed in Section 4.

\section{Main Result}

In this section, we prove a reciprocal relation giving $y_{1-r}$ in terms of multivariate Hermite polynomials. The exponential generating function (egf) for $H_{\mathbf{k}}=H_{\mathbf{k}}(\mathbf{x}, \mathbf{V})$ is

$$
\sum_{\mathbf{k} \geq \mathbf{0}_{p}} H_{\mathbf{k}} \mathbf{t}^{\mathbf{k}} / \mathbf{k} !=\mathbb{E}\left[e^{\mathbf{t}^{\prime}(\mathbf{y}+\mathrm{i} \mathbf{Y})}\right]=e^{\mathbf{t}^{\prime} \mathbf{y}} \mathbb{E}\left[e^{i \mathbf{t}^{\prime} \mathbf{Y}}\right]
$$

for $\mathbf{t}, \mathbf{x} \in \mathbb{R}^{p}$. The coefficients of $\mathbf{t}^{\mathbf{k}}$ in these three terms are $H_{\mathbf{k}} / \mathbf{k} !, \mathbf{y}^{\mathbf{k}} / \mathbf{k}$ ! and $\mathbb{E}\left[(\mathrm{i} Y)^{\mathbf{k}}\right] / \mathbf{k}$ !. So, taking the coefficient of $\mathbf{t}^{\mathbf{k}}$ gives

$$
H_{\mathbf{k}} / \mathbf{k} !=\mathbf{y}^{\mathbf{k}} / \mathbf{k} ! \otimes \mathbb{E}\left[(\mathrm{iY})^{\mathbf{k}}\right] / \mathbf{k} !
$$

which implies (1.2), where

$$
a_{\mathbf{r}} \otimes b_{\mathbf{r}}=\sum_{\mathbf{0}_{p} \leq \mathbf{k} \leq \mathbf{r}} a_{\mathbf{k}} b_{\mathbf{r}-\mathbf{k}},
$$

the discrete multivariate convolution. Substituting (1.1) gives the result of [10]. For $\mathbf{t} \in \mathbb{R}^{p}$,

$$
1=e^{\mathbf{t}^{\prime} \mathbf{V}^{-1} \mathbf{t} / 2} e^{-\mathbf{t}^{\prime} \mathbf{V}^{-1} \mathbf{t} / 2}=\mathbb{E}\left[e^{\mathbf{t}^{\prime} \mathbf{Y}}\right] \mathbb{E}\left[e^{i \mathbf{t}^{\prime} \mathbf{Y}}\right] .
$$

Multiplying by $e^{\mathbf{t}^{\prime} \mathbf{y}}$ gives

$$
e^{\mathbf{t}^{\prime} \mathbf{y}}=\mathbb{E}\left[e^{\mathbf{t}^{\prime} \mathbf{y}+\mathbf{i} \mathbf{t}^{\prime} \mathbf{Y}}\right] \mathbb{E}\left[e^{\mathbf{t}^{\prime} \mathbf{Y}}\right] .
$$

Therefore

$$
\mathbf{y}^{\mathbf{r}} / \mathbf{r} !=H_{\mathbf{r}} / \mathbf{r} ! \otimes \mathbb{E}\left[\mathbf{Y}^{\mathbf{r}}\right] / \mathbf{r} !
$$

and hence

$$
\mathbf{y}^{\mathbf{r}}=\sum_{\mathbf{0}_{p} \leq \mathbf{k} \leq \mathbf{r}} H_{\mathbf{k}} \mathbb{E}\left[\mathbf{Y}^{\mathbf{r}-\mathbf{k}}\right] .
$$

As a result, we can swap $H_{\mathbf{k}}$ and $\mathbf{y}^{\mathbf{k}}$ in (1.2) if we make all signs positive. The same is true if we use their super forms. 


\section{The Modified Hermite Polynomials}

Closely related are the modified Hermite polynomials. In the univariate case these were introduced by Fisher [5]. They are

$$
H_{k}^{*}(x)=e^{-x^{2} / 2} D^{k} e^{x^{2} / 2}=\mathrm{i}^{-k} H_{k}(\mathrm{i} x)=\mathrm{i}^{k} H_{k}(-\mathrm{i} x)
$$

for $k \geq 0, D=d / d x$, and $x \in \mathbb{R}$. That is, $H_{k}^{*}=H_{k}^{*}(x)$ is $H_{k}(x)$ with all its signs made positive. Withers and McGavin [11] gave the simpler formula

$$
H_{k}^{*}=\mathbb{E}\left[(x+N)^{k}\right], k \geq 0,
$$

and other formulas for it, and applied to find $\mathbb{E}\left[(x+N)^{-n}\right], n \geq 0$. For applications to repeated integrals of the univariate normal density, see Withers and Nadarajah $[12,13,14]$.

We define the modified multivariate Hermite polynomial in sub form as

$$
\begin{aligned}
H_{\mathbf{k}}^{*} & =H_{\mathbf{k}}^{*}(\mathbf{x}, \mathbf{V})=\phi_{\mathbf{V}}(\mathbf{x})\left(-\partial_{\mathbf{x}}\right)^{\mathbf{k}} \phi_{\mathbf{V}}(\mathbf{x})^{-1} \\
& =\mathbb{E}\left[(\mathbf{y}+\mathbf{Y})^{\mathbf{k}}\right]=\sum_{\mathbf{0}_{p} \leq \mathbf{j} \leq \mathbf{k}}\left(\begin{array}{l}
\mathbf{k} \\
\mathbf{j}
\end{array}\right) \mathbf{y}^{\mathbf{k}-\mathbf{j}} \mathbb{E}\left[\mathbf{Y}^{\mathbf{j}}\right]
\end{aligned}
$$

for $\mathbf{x} \in \mathbb{R}^{p}, \mathbf{y}=\mathbf{V}^{-1} \mathbf{x}$ and $\mathbf{k} \in \mathbb{N}^{p}$. We define multivariate Hermite polynomial in super form as

$$
\begin{aligned}
H_{*}^{1-r} & =H_{*}^{j_{1}, \ldots, j_{r}}(\mathbf{x}, \mathbf{V})=\phi_{\mathbf{V}}(\mathbf{x})\left(-\partial_{j_{1}}\right) \cdots\left(-\partial_{j_{r}}\right) \phi_{\mathbf{V}}(\mathbf{x})^{-1} \\
& =\mathbb{E}\left[\prod_{s=1}^{r}(\mathbf{y}+\mathbf{Y})_{j_{s}}\right]=\sum_{0 \leq s \leq r / 2} \sum_{\left(\begin{array}{c}
r \\
s
\end{array}\right)} y_{1-(r-2 s)} V^{(r-2 s+1)-r} \\
& =y_{1-r}+\sum_{\left(\begin{array}{c}
r \\
2
\end{array}\right)} y_{1-(r-2)} V^{(r-1)-r}+\sum_{\left(\begin{array}{c}
r \\
4
\end{array}\right)} y_{1-(r-4)} V^{(r-3)-r}+\cdots
\end{aligned}
$$

That is, (3.4) is the multivariate Hermite polynomial with all signs made positive. We have not seen (3.4) before in the literature. They should be useful for extending the applications of $H_{n}^{*}(x)$ in Fisher [5] and Withers and Nadarajah $[\mathbf{1 2}, \mathbf{1 3}, \mathbf{1 4}]$ to their multivariate versions. $H_{\mathbf{k}}^{*}$ has egf

$$
\sum_{\mathbf{k} \geq \mathbf{0}_{p}} H_{\mathbf{k}}^{*} \mathbf{t}^{\mathbf{k}} / \mathbf{k} !=\mathbb{E}\left[e^{\mathbf{t}^{\prime}(\mathbf{y}+\mathbf{Y})}\right]=e^{\mathbf{t}^{\prime} \mathbf{y}} \mathbb{E}\left[e^{\mathbf{t}^{\prime} \mathbf{Y}}\right]
$$

for $\mathbf{t}, \mathbf{x} \in \mathbb{R}^{p}$. By a similar argument to that in Section 2, the reciprocal relation holds; that is, one can swap $H_{\mathbf{k}}^{*}$ and $\mathbf{y}^{\mathbf{k}}$ in (3.3) if signs are made to alternate. Similarly, one can swap $H_{*}^{1-r}$ and $y_{1-r}$ in (3.4) if signs are made to alternate.

\section{Extensions}

A natural question is whether $H_{k}(x)=\mathbb{E}\left[(x+\mathrm{i} N)^{k}\right]$ can be extended for random variables other than the unit normal random variable. Nadarajah [7] showed that if $Z$ is a symmetric stable random variable then $\mathbb{E}\left[(x+\mathrm{i} Z)^{k}\right]$ is a generalized Hermite polynomial due to Djordjevic [4]. Nadarajah [8] showed that if $Z$ is a Student's $t$ random variable then $\mathbb{E}\left[(x+\mathrm{i} Z)^{k}\right]$ is a modified Chebyshev polynomial. 
However, such representations for general random variables do not appear to be possible. For example, if $Z$ is a uniform $[-a, a]$ random variable then

$$
\mathbb{E}\left[(x+\mathrm{i} Z)^{k}\right]=\frac{(x+a \mathrm{i})^{k+1}-(x-a \mathrm{i})^{k+1}}{2 a \mathrm{i}(k+1)} .
$$

\section{Acknowledgements}

The authors thank the Editor and the referee for careful reading and comments which greatly improved the paper.

\section{References}

[1] Y., Ait-Sahalia, Closed-form likelihood expansions for multivariate diffusions, Ann. Stat. 36 (2) (2008), 906-937.

[2] R. N. Bhattacharya and J. K. Ghosh, On the validity of the formal Edgeworth expansion, Ann. Stat. 6 (1978), 434-451.

[3] E., Di Nardo, G. Guarino and D. Senato, A new algorithm for computing the multivariate Faa di Bruno's formula, Appl. Math. Comput. 217 (13) (2011), 6286-6295.

[4] G. Djordjevic, On some properties of generalized Hermite polynomials, Fibonacci Q. 34 (1) (1996), 2-6.

[5] R. A. Fisher, Introduction to Table of Hh Functions, British Association for the Advancement of Science, xxiv-xxxiv, 1931.

[6] S. Fouques, D. Myrhaug, and F. G. Nielsen, Seasonal modeling of multivariate distributions of Metocean parameters with application to marine operations, J. of Offshore Mechanics and Arctic Engineering 126 (2004), 202-212.

[7] S. Nadarajah, Simple formulas for certain polynomials, Appl. Math. Comput. 187 (2) (2007), 1592-1596.

[8] S. Nadarajah, On modified Chebyshev polynomials, J. Math. Anal. Appl. 334 (2) (2007), 1492-1494.

[9] S. M. Kendall, A. Stuart and J. K. Ord, Kendall's Advanced Theory of Statistics. Volume 1: Distribution Theory, fifth edition, Charles Griffin \& Company, London, 1987

[10] C. S. Withers, A simple expression for the multivariate Hermite polynomials, Stat. Probab. Lett. 47 (2) (2000), 165-169.

[11] C. S. Withers and P. McGavin, Expressions for the normal distribution and repeated normal integrals, Stat. Probab. Lett. 76 (5) (2006), 479-487.

[12] C. S. Withers and S. Nadarajah, New expressions for repeated lower tail integrals of the normal distribution, J. Korean Stat. Soc. 36 (3) (2007), 411-421.

[13] C. S. Withers and S. Nadarajah, New expressions for repeated upper tail integrals of the normal distribution, Methodol. Comput. Appl. Probab. 13 (4) (2011), 855-871.

[14] C. S. Withers and S. Nadarajah, Repeated integrals of the univariate normal as a finite series with the remainder in terms of Moran's functions, Statistics 46 (1) (2012), 13-22.

[15] C. S. Withers and S. Nadarajah, The dual multivariate Charlier and Edgeworth expansions, Stat. Probab. Lett. 87 (2014), 76-85. 
[16] X. Zhang, Y. Zhang, M. D. Pandey and Y. Zhao, Probability density function for stochastic response of non-linear oscillation system under random excitation, International J. of Non-Linear Mechanics 45, 800-808.

Christopher S. Withers

Callaghan Innovation,

Lower Hutt,

New Zealand

kit.withers@gmail.com
Saralees Nadarajah

University of Manchester,

Manchester M13 9PL,

UK

mbbsssn2@manchester.ac.uk 